\title{
ORIGINAL
}

\section{Sex-based differences in ED management of critically ill patients with sepsis: a nationwide cohort study}

Jonas Sunden-Cullberg ${ }^{1^{*}} \mathbb{0}$, Anton Nilsson ${ }^{2}$ and Malin Inghammar ${ }^{3,4^{*}}$

(c) 2020 The Author(s)

\begin{abstract}
Purpose: To compare management and outcomes for critically ill women and men with sepsis in the emergency medical services (EMS), the emergency department (ED) and the ICU.

Methods: We used two prospectively compiled Swedish national quality registers, the National Quality Sepsis Registry and the Swedish Intensive Care Registry to identify a nationwide cohort of 2720 adults admitted to an ICU within $24 \mathrm{~h}$ of arrival to any of 32 EDs, with a diagnosis of severe sepsis or septic shock between 2008 and 2015.

Results: Patients were $44.5 \%$ female. In the EMS, a higher fraction of men had all vital signs recorded - 54.4 vs $49.9 \%$ ( $p=0.02$ ) and received IV fluids and oxygen -40.0 vs $34.8 \%(p=0.02)$. In the ED, men had completed 1-h sepsis bundles in $41.5 \%$ of cases compared to $30.0 \%$ in women ( $p<0.001)$, and shorter time to antibiotics -65 (IQR 30-136) vs 87 min (IQR 39-172) ( $p=0.0001)$. There was no significant difference between men and women regarding ICU nursing workload, mechanical ventilation or ICU length of stay. In severity-adjusted multivariable analysis, OR for women achieving a completed sepsis bundle, compared to men was 0.64 (Cl 0.51-0.81). Thirty-day mortality was $25.0 \%$ for women and $23.1 \%$ for men $(p=0.24)$. Adjusted OR for female death was 1.28 (Cl 1.00-1.64), but the increased mortality was not mediated by differential bundle completion.
\end{abstract}

Conclusions: Women and men with severe sepsis or septic shock received differential care in the ED, but this did not explain higher odds of death in women.

Keywords: Sepsis, Sex, Mortality, Management, ICU, ED, Emergency medical services

*Correspondence: jonas.sunden-cullberg@ki.se; malin.inghammar@med. lu.se

${ }^{1}$ Division of Infectious Diseases I 73 and Center for Infectious Medicine, Karolinska Institutet at Karolinska University Hospital Huddinge, 141 86 Stockholm, Sweden

${ }^{4}$ Department of Infectious Diseases Lund, Skåne University Hospital, Hälsogatan 3, SE-221 85 Lund, Sweden

Full author information is available at the end of the article

\section{Introduction}

Women receive differential care compared to men in coronary heart disease [1, 2], stroke [3], cancer [4], joint replacements [5], trauma [6], and other medical conditions. In the ICU, men are more likely to receive invasive procedures such as mechanical ventilation and catheterization [7-9], incur higher nurse workload, and receive longer ICU stays [10].

Sepsis contributes to one in every two to three hospital deaths [11]. Among the few interventions associated with improved survival are short time to antibiotics [12-15], and completion of sepsis bundles [13-16].

\section{望 Springer}


The impact of patient sex on management of sepsis has been sparsely investigated. In a previous small study on severe sepsis and septic shock, we observed that men received antibiotics more rapidly than women [17]. We have identified only one single-center study systematically focused on the influence of sex on the management of critically ill septic patients in the ED $[18,19]$. It found that men received antibiotics more promptly than women. Reports on sex and mortality from sepsis have shown conflicting results $[9,18,20-25]$.

The aim of this multicenter study was to investigate if men and women with community-acquired severe sepsis or septic shock received differential management in the emergency medical services (EMS), the emergency department (ED) or the ICU; whether mortality differed and; if differential management in the ED explains differences in mortality.

\section{Methods}

\section{Study design}

We created a historical cohort based on all patients registered in the prospectively compiled National Quality Sepsis Registry (NQSR) [26] with concurrent registration in the Swedish Intensive Care Registry (SIR) www. icuregswe.org [27]. The NQSR comprises patients, aged 18 and over, admitted to an ICU with a diagnosis of community-acquired severe sepsis or septic shock within $24 \mathrm{~h}$ of arrival to an ED [26]. In NQSR, infectious disease (ID) specialists at each site screen hospital records. Eligible patients are entered into the NQSR database. The SIR currently covers all general ICUs in Sweden but has not had complete coverage during the study period. This report includes patients registered in NQSR from January 2008 until December 2015 and follow-up on 30-day mortality until data extraction on March 1 2016. Data on NQSR patients were then extracted from SIR. Additional information on comorbidity was obtained from the Swedish National Inpatient Register [28] and the Swedish Prescribed Drug Register [29].

Data collection, exclusion criteria, definitions and variables Severe sepsis and septic shock were diagnosed using a modified version of the 1992 sepsis definition [30], in practice accepting a diagnosis of severe sepsis on the basis of infection + organ dysfunction (Online supplement). Processes of care in the EMS, ED and ICU are listed in Table 2, including sepsis bundle and time to antibiotics as the main variables of interest. The sepsis bundle required: IV fluids; lactate/base excess measured; blood cultures before antibiotics and; administered antibiotics-all within $1 \mathrm{~h}$. A composite nursing workload score was built from quartiles of three nursing workload scores. For further details on data collection, variables

\section{Take-home message}

In this nationwide cohort study of adults with severe sepsis or septic shock admitted via the emergency department within $24 \mathrm{~h}$ to the ICU, sepsis bundle completion was lower and time to antibiotics slower in women compared to men. Women had higher adjusted odds of dying, but the increase was not mediated by differential bundle completion.

in NQSR and SIR and definitions, including SAPS3 and tSAPS3, see Online supplement.

\section{Cohort}

By December 2015, 3240 patients were recorded in NQSR. Of these, 520 were excluded yielding 2720 in the final cohort, Fig. 1. Patients were admitted via 32 EDs to 42 ICUs; 904 in 7 university hospitals and 1816 patients in 25 county hospitals.

\section{Statistical methods}

Chi square and Wilcoxon rank sum tests were used to assess the distribution of risk factors for sepsis bundle completion and 30-day mortality between men and women. Logistic regression was used to estimate odds ratios (ORs) for sepsis bundle completion and 30-day mortality. Generalized estimating equations (GEEs) were used to account for the possibility of dependency between individuals admitted through the same ED. In the analysis of associations between sex and ED processes of care, we selected a priori standard clinical variables known or expected to be potential confounders of the sex quality of care association: arrival by ambulance [31]; age; vital signs (generally used for triage scoring); comorbidities; and suspected infectious focus. The selected covariates were assessed in several multivariable models, representing successive decision points in the management of a septic patient, from initial triage using just age and mode of arrival, adding vital signs, past medical history and finally suspected diagnosis for the main model. As an auxiliary analysis, we adjusted for SAPS3, which is not calculated until ICU admittance, up to $24 \mathrm{~h}$ after ED triage, but is the best global measure of severity in the database, and a potential confounder. ORs were also estimated in subgroups of participants classified according to age, body temperature, Charlson score, SAPS3 score, year of inclusion and hospital type. Homogeneity of estimates across subgroups was assessed using likelihood ratio tests.

Among risk factors for mortality, we similarly selected a priori potential confounders to the sex-mortality association. SAPS3 was used as measure of disease severity alongside body temperature, which we have shown to be an important determinant of outcome [32] and 


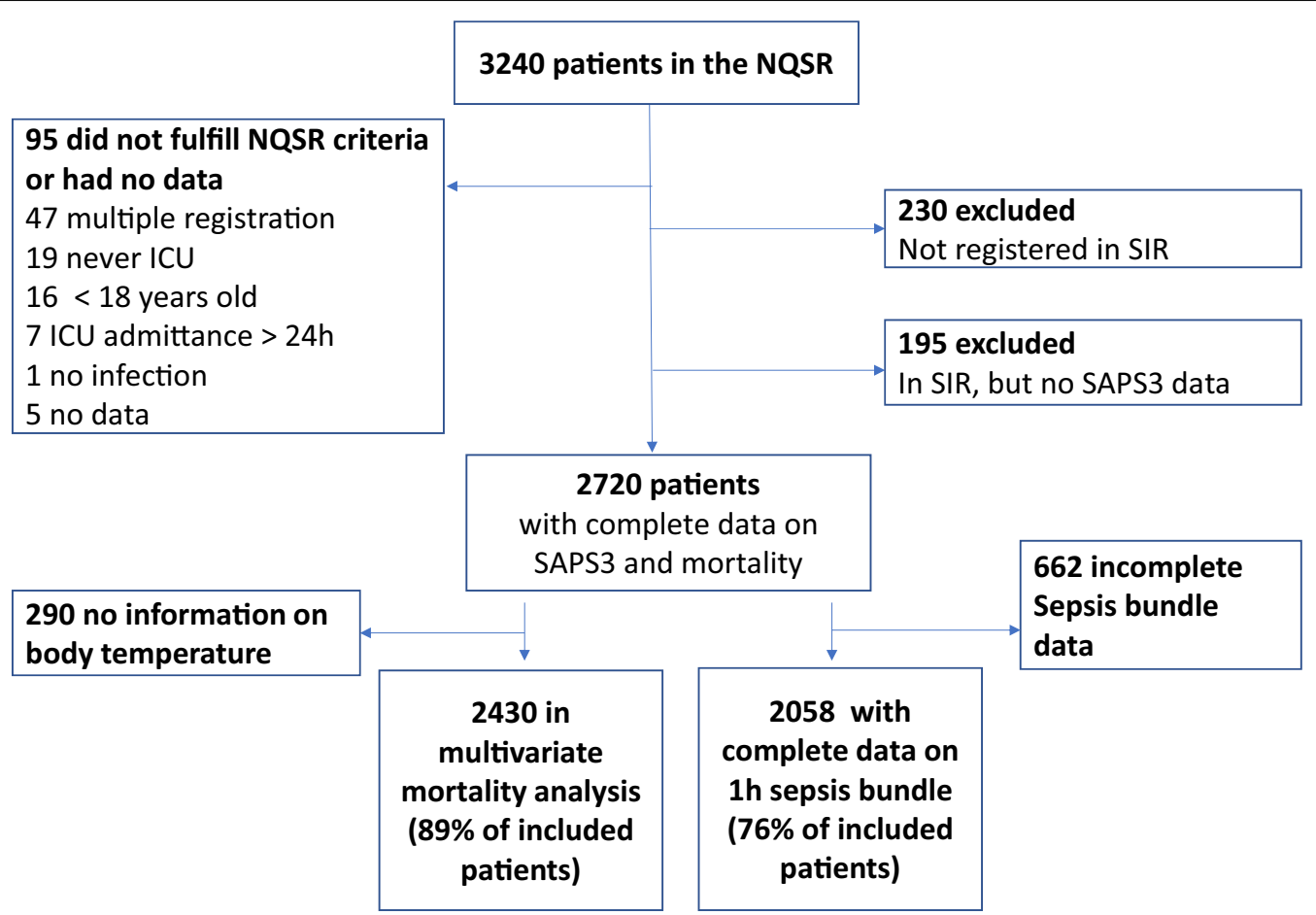

Fig. 1 Study flow chart. NQSR National Quality Sepsis Registry, SIR Swedish Intensive Care registry, ICU Intensive Care unit. Ninety-five patients were excluded because they did not fulfill NQSR inclusion criteria or had no data registered. 230 were excluded since they were not registered in SIR and 195 patients since a different severity score was used in the ICU and they lacked SAPS3 data. For transparency, baseline variables, outcomes and quality of care for the latter two groups minus 14 patients for whom 30-day mortality was not registered are shown in Tables 1 and 2

which differs in men and women. Treatment restrictions and incorrect antibiotics were also deemed important risk factors and possible confounders. In an additional model, infectious diagnoses and Charlson comorbidities not included in SAPS3 were added. A directed acyclic graph shows the hypothesized relationships between different types of variables in our analyses, Online supplement figure e1. Analyses were performed in models with and without treatment in the form of completed sepsis bundles. Mediation of the effects of sex on mortality by bundle fulfillment and SAPS3 score was evaluated using Stata's paramed command. Stata version 15 was used for statistical analysis (StataCorp, College Station, TX).

\section{Results}

The final cohort included 2720 patients. Table 1 shows patient characteristics and outcomes and, for transparency, data from 411 patients excluded since they were not registered in SIR or lacked SAPS3 data. Females constituted $44.5 \%$ of patients and median age was 68 for both sexes. Men had marginally higher median SAPS3 score65 vs 64 , and higher Charlson score $-2.56( \pm 2.35$, mean $\mathrm{SD})$ vs $2.12( \pm 2.11$, mean $S D)$. Higher severity among men was confined to patients under $60-$ SAPS3 59 vs 55 , whereas severity did not differ in the age cohorts 60-69
(SAPS3 63), 70-79 (69), and 80 and over (71). Thirtyday mortality was $25.0 \%$ for women and $23.1 \%$ for men $(p=0.24)$. In those aged 50 and under $(n=453)$, mortality was $6 \%$ for women and $10 \%$ for men $(p=0.13)$. At all cutoffs over the age of 50, mortality was higher among women: $>50(n=2267) 29$ vs $25 \%(p=0.05) ;>60$ $(n=1881) 31$ vs $27 \%(p=0.02)$; $>70(n=1175) 37$ vs $31 \%$ $(p=0.02) ;>80(n=479), 46$ vs $38 \%(p=0.06)$.

\section{Sex and differential care}

Table 2 shows crude process of care variables. In the EMS, more men had all vital signs recorded and received IV fluids and oxygen. In the ED, men had complete $1 \mathrm{~h}$ sepsis bundles in $41.5 \%$ of cases compared to $30.0 \%$ in women $(p<0.001)$, and shorter time to antibiotics -65 (IQR 30-136) vs $87 \mathrm{~min}$ (IQR 39-172) ( $p=0.0001)$. ICU nursing workload, mechanical ventilation and length of stay were equally distributed.

Figure 2 shows sepsis bundle completion and time to antibiotics stratified according to age, suspected site of infection, comorbidity, SAPS3 score, respiratory rate and body temperature. Men had higher sepsis bundle completion and shorter time to antibiotics in almost all strata, while the reverse did not occur in any stratum. 
Table 1 Patient characteristics and outcomes

\begin{tabular}{|c|c|c|c|c|c|}
\hline Characteristic & All patients & Women & Men & Excluded women & Excluded men \\
\hline Number of patients & 2720 & $1210(44.5 \%)$ & $1510(55.5 \%)$ & 172 & 239 \\
\hline \multicolumn{6}{|l|}{ Demography } \\
\hline Age, median, IQR & $68,57-77$ & $68,56-77$ & $68,58-77$ & $69,55-78$ & $69,58-78$ \\
\hline Severity of disease-SAPS3 & $64,56-74$ & $64,55-73$ & $65,56-75$ & - & - \\
\hline \multicolumn{6}{|l|}{ Underlying comorbidity } \\
\hline CCI, median, IQR (Mean, SD) & $2,1-4(2.37 \pm 2.25)$ & $2,0-3(2.12 \pm 2.11)$ & $2,1-4(2.56 \pm 2.35)$ & - & \\
\hline \multicolumn{6}{|l|}{ Focus of infection (\% of patients) } \\
\hline Pneumonia & 33.8 & 30.9 & 36.1 & 36.1 & 35.0 \\
\hline Urinary tract & 20.5 & 21.3 & 19.9 & 20.9 & 20.7 \\
\hline Abdominal infection & 11 & 11.9 & 10.2 & 14 & 13.1 \\
\hline Other focus & 21.5 & 21.9 & 21.3 & 15.1 & 20.7 \\
\hline Unknown ( $n=308)$ and missing $(n=51)$ & 13.2 & 14 & 12.6 & 14 & 10.6 \\
\hline \multicolumn{6}{|l|}{ Etiology (\%) } \\
\hline Gram-positive, $n=965$ & 35 & 35 & 35 & 37 & 35 \\
\hline Gram-negative, $n=871$ & 32 & 33 & 31 & 36 & 34 \\
\hline Other, $n=119$ & 4 & 4 & 5 & 3 & 3 \\
\hline Unknown, $n=705$ and missing, $n=60$ & 28 & 27 & 29 & 24 & 28 \\
\hline \multicolumn{6}{|l|}{ Vital signs, $\%$ of patients and lactate } \\
\hline $\mathrm{BT},{ }^{\circ} \mathrm{C}^{\mathrm{a}}$, median, IQR & $38.1,37-39.1$ & $38,37-39$ & $38.2,37-39.1$ & $38.2,37-39$ & $38.2,37-39$ \\
\hline SBP, mm Hg ${ }^{b}$, median, IQR & $110,90-131$ & $108,90-130$ & $110,90-132$ & $106,88-133$ & $105,86-132$ \\
\hline $\mathrm{SBP}<90 \mathrm{~mm} \mathrm{Hg}$ & 23.1 & 23.7 & 22.7 & 26.1 & 28.1 \\
\hline $\mathrm{RR}>20 / \mathrm{min}^{\mathrm{c}}$ & 77.4 & 74 & 80 & 82.1 & 81.1 \\
\hline Saturation $<90 \%{ }^{\mathrm{d}}$ & 26.8 & 27.9 & 25.9 & 33.8 & 37.8 \\
\hline $\mathrm{RLS}>1^{\mathrm{e}}$ & 25.1 & 25.2 & 25 & 29.7 & 31.8 \\
\hline $\mathrm{PR}>90 \mathrm{bpm}^{\mathrm{f}}$ & 75.6 & 76.2 & 75.1 & 77.6 & 74.8 \\
\hline $\begin{array}{l}\text { Lactate } \mathrm{mmol} / \mathrm{L} \text {, median, IQR }{ }^{9} \text { median, } \\
\text { IQR }\end{array}$ & $3.6,2.1-5.9$ & $3.7,2.2-5.9$ & $3.5,2.5-5.8$ & $3,1.8-5$ & $3,1.8-5$ \\
\hline \multicolumn{6}{|l|}{ Patient outcomes } \\
\hline 30-day mortality, \% & 24 & 25 & 23.1 & 22.1 & 22.2 \\
\hline LOS, survivors ICU, hours & $54,26-135$ & $57,28-140$ & $52,25-131$ & - & - \\
\hline LOS, survivors hospital, days & $14,8-26$ & $15,8-26$ & $13,8-25$ & $9,5-19$ & $9,4-21$ \\
\hline
\end{tabular}

$B T$ body temperature, $C C I$ Charlson comorbidity index, $I Q R$ interquartile range, $L O S$ length of stay, $P R$ pulse rate, $R L S$ reaction level scale $(1=$ normal, $>1=$ affected consciousness), RR Respiratory rate, SAPS3 Simplified Acute Physiology Score 3, SBP systolic blood pressure, SD standard deviation, $h$ hours, $d$ days. Excluded patients = patients who were not registered in SIR or who lacked SAPS3 data. Vital signs and lactate measured on ED admittance

a Data missing for 290 patients (11\%)

b Data missing for 69 patients (3\%)

c Data missing for 210 patients (8\%)

d Data missing for 107 patients (4\%)

e Data missing for 479 patients (18\%)

f Data missing for 79 patients (3\%)

9 Data missing for 755 patients (28\%)

\section{Sepsis bundle completion}

Figure e2 shows OR for sepsis bundle completion in women compared to men in univariate and different multivariable models, representing available information at successive management decision points in the ED. In the main model, OR for women achieving a completed sepsis bundle, compared to men, was 0.64 (CI 0.51-0.81), adjusted for mode of arrival to the ED, age, vital signs, comorbidities and suspected infectious focus. In an auxiliary analysis, we adjusted for SAPS3 score, which yielded an OR of $0.59(0.50-0.70)$. Over all different models, ORs for women achieving a completed sepsis bundle varied within a narrow range of $0.57-0.64$.

\section{Subgroup analyses}

We observed no statistical support for heterogeneity in the ORs of bundle fulfillment by sex according to 
Table 2 Processes of care in women and men

\begin{tabular}{|c|c|c|c|c|c|c|c|}
\hline Characteristic & All patients & Women & Men & $\begin{array}{l}\text { Women vs men } \\
p \text { value }\end{array}$ & Excluded women & Excluded men & $\begin{array}{l}\text { Excluded } \\
\text { women vs } \\
\text { men } \\
p \text { value }\end{array}$ \\
\hline Number of patients & 2720 & $1210(44.5 \%)$ & $1510(55.5 \%)$ & & 172 & 239 & \\
\hline \multicolumn{8}{|l|}{ Processes of care, $\%$ of patients } \\
\hline \multicolumn{8}{|l|}{ EMS } \\
\hline Arrival in ambulance ${ }^{a}$ & 80.6 & 79.7 & 81.3 & 0.3 & 79.3 & 82.9 & 0.4 \\
\hline $\begin{array}{l}\text { Saturation, SBP, RR, PR all meas- } \\
\text { ured and recorded in ambu- } \\
\text { lance }\end{array}$ & 52.4 & 49.9 & 54.4 & 0.02 & 47.8 & 46.7 & 0.8 \\
\hline $\begin{array}{l}\text { Oxygen and IV fluids in ambu- } \\
\text { lance }\end{array}$ & 37.7 & 34.8 & 40 & 0.02 & 23.5 & 41.2 & 0.002 \\
\hline \multicolumn{8}{|l|}{ Emergency department } \\
\hline $\begin{array}{l}\text { All vital signs measured and } \\
\text { recorded in ED }\end{array}$ & 61.8 & 60.9 & 62.6 & 0.37 & 59.9 & 59.9 & 1 \\
\hline $\begin{array}{l}\text { Time to } A B^{b} \text { median, } \\
I Q R\end{array}$ & $\begin{array}{l}75 \min \\
34-150\end{array}$ & $\begin{array}{l}87 \min \\
39-172\end{array}$ & $\begin{array}{l}65 \mathrm{~min} \\
30-136\end{array}$ & 0.0001 & $\begin{array}{l}81 \mathrm{~min} \\
46-200\end{array}$ & $\begin{array}{l}61 \mathrm{~min} \\
25-130\end{array}$ & 0.003 \\
\hline $\mathrm{AB}$ within $1 \mathrm{~h}$ & 43.6 & 38.6 & 47.6 & $<0.001$ & 39.7 & 50.0 & 0.05 \\
\hline$A B$ within $3 \mathrm{~h}$ & 80.6 & 77.1 & 83.5 & $<0.001$ & 73.7 & 85.9 & 0.004 \\
\hline Iv fluids within $1 \mathrm{~h}^{\mathrm{c}}$ & 82.3 & 80.9 & 83.5 & 0.08 & 81.3 & 86.3 & 0.3 \\
\hline Lactate/BE measured < $1 \mathrm{~h}$ & 77.4 & 75.2 & 79.1 & 0.02 & 65.7 & 76.0 & 0.02 \\
\hline$B C$ before $A B^{d}$ & 93.6 & 91.8 & 95.1 & 0.001 & 94.5 & 95.4 & 0.8 \\
\hline Lactate within $1 \mathrm{~h}$ & 70.4 & 68.4 & 72 & 0.09 & 60 & 68.8 & 0.1 \\
\hline Bundle $1 h^{e}$ & 36.4 & 30 & 41.5 & $<0.001$ & 31.3 & 45.4 & 0.05 \\
\hline ICU & & & & & - & - & \\
\hline $\begin{array}{l}\text { Composite NWS median, IQR } \\
(\text { mean, SD) }\end{array}$ & $\begin{array}{l}2,1-3 \\
(2.35 \pm 1.14)\end{array}$ & $\begin{array}{l}2,1-3 \\
(2.36 \pm 1.14)\end{array}$ & $\begin{array}{l}2,1-3 \\
(2.35 \pm 1.15)\end{array}$ & 0.84 & - & - & \\
\hline Mechanical ventilation & 30.1 & 29.3 & 30.7 & 0.43 & - & - & \\
\hline Incorrect $A B$ & 7.5 & 7.1 & 7.8 & 0.49 & 3.5 & 4.2 & 0.7 \\
\hline Treatment limitations $48 \mathrm{~h}$ & 20.2 & 20.2 & 20.2 & 0.98 & 12.8 & 15.2 & 0.5 \\
\hline
\end{tabular}

$A B$ antibiotics, $B C$ blood culture, $B E$ base excess, $B T$ body temperature, Bundle $1 \mathrm{~h}$ completed sepsis bundle within one hour, $E M S$ emergency medical services, $E D$ emergency department, IQR interquartile range, NWS nursing workload score, $P R$ pulse rate, $R R$ respiratory rate, $S B P$ systolic blood pressure, $S D$ standard deviation. Excluded patients $=$ patients who were not registered in SIR or who lacked SAPS3 data

a Data missing for 85 patients (3\%)

b Data missing for 311 patients (11\%)

c Data missing for 228 patients (8\%)

d Data missing for 336 patients (12\%)

e Complete data missing for 662 patients (24\%)

$f$ Data missing for 52 patients (2\%)

different subgroups of age $(p=0.42)$, body temperature $(p=0.33)$, comorbidity $(p=0.60)$, severity $(p=0.84)$, year of inclusion $(p=0.15)$ or tertiary/secondary hospitals $(p=0.12)$, in models adjusted for mode of arrival to the ED, age, vital signs, body temperature, Charlson score and preliminary focus of infection. The stratum-specific ORs are shown in Online supplement Table e1.

\section{Sex and mortality}

Table 3 shows ORs for individual risk factors and from multivariable analyses for 30-day mortality. In crude analysis, the OR for death in women compared to men was 1.11 (CI 0.91-1.36). In multivariable analysis, the OR was 1.28 (CI 1.00-1.64) adjusted for tSAPS3, body temperature, incorrect antibiotics and treatment limitations. The estimated absolute risk difference was 31 (CI 0-62) additional female deaths per 1000 ICU admissions. Addition to the model of treatment in the form of completed sepsis bundles led to the loss of 548 patients due to missing values; the OR estimate remained unchanged at 1.28 (CI 0.97-1.70). There was no association between bundle completion and mortality in either uni- or multivariable 
(See figure on next page.)

Fig. 2 a, c, e, $\mathbf{g}, \mathbf{i}$ and $\mathbf{k}$ (left column) shows fraction of patients who achieved a 1-h sepsis bundle stratified by sex and $\mathbf{a}$ age $(n=2058)$, $\mathbf{c}$ diagnoses $(n=2058)$, e Charlson comorbidity index $(n=2058), \mathbf{g}$ SAPS3 quartiles $(n=2058)$, i respiratory rate $(n=1936)$, and $\mathbf{k}$ body temperature $(n=1882)$. Figure $1 \mathrm{~b}(n=2409), \mathrm{d}(n=2409), \mathrm{f}(n=2409), \mathrm{h}(n=2409), \mathrm{j}(n=2259)$ and $\mathrm{I}(n=2191)$ (right column) were similarly stratified to show time to antibiotics. ${ }^{*}$ Indicates $p$ value $<0.05,{ }^{* *}<0.01,{ }^{* * *}<0.001$ and ${ }^{* * * *}<0.0001$. Indicates a value between 0.05 and 0.1 and $n .5$ indicates nonsignificant. $A B$ antibiotics, Pneum pneumonia, UTI urinary tract infection, Abdom abdominal infection, Other other focus of infection, Unkn unknown focus of infection, Ch Charlson score, SAPS3 Severe Acute Physiology Score 3, RR respiratory rate, Temp body temperature

analysis. Further addition of infectious diagnoses and Charlson comorbidities not included in SAPS3 yielded an OR of 1.29 (CI 0.97-1.71). The increase from lower OR in univariate to higher in multivariable analysis is explained by the addition of tSAPS3 which alone increased OR for female death from 1.11 to 1.26 (CI 1.01-1.57). The major contribution was from non-physiologic SAPS3 subscores-OR was $1.22(0.98-1.52)$ when vital signs and laboratory SAPS3 subscores were subtracted.

A formal mediation analysis, table e2, Online supplement, showed that the effects of sex on mortality could not be explained by bundle completion; the odds ratio for the indirect effect of being female was 0.99 (CI $0.91-$ 1.07), whereas the direct effect of 1.26 (CI 0.99-1.60) was similar to the total effect of $1.24(0.97-1.59)$. The absence of mediation by bundle completion is in line with the result that bundle completion does not decrease mortality. Examining SAPS3 as a mediator, we found evidence of both an indirect and direct effect; the direct effect amounted to 1.25 (CI 1.03-1.51) and the indirect 0.91 (0.85-0.98), quantifying the opposing consequences of female sex-beneficial in terms of disease severity and adverse in terms of other factors. Note that in the analysis considering SAPS3 as a mediator, sex is interpreted as biological sex, whereas in the other analyses sex is interpreted as gender (as observed by healthcare staff), and severity is then considered a confounder.

Adjusted OR for female death did not significantly differ between those who did and did not receive 1 -h bundle completion, $1.28(0.81-2.03)$ vs $1.29(0.95-1.77), p=0.86$, or among those with and without treatment restrictions, $1.42(0.99-2.05)$ vs $1.24(0.94-1.66), p=0.48$.

\section{Discussion}

\section{Main findings}

In this nationwide cohort study, we compared management in the EMS, ED and ICU of women and men admitted to an ICU with community-acquired severe sepsis or septic shock. In the ED, a 1-h sepsis bundle was fulfilled $38 \%$ more often and median time to antibiotics was $34 \%$ faster in men than in women. The findings were consistent in nearly every subgroup, whichever way we stratified data, and in multivariable analyses adjusted for potential confounders. Using models representing successive clinical decision points, we showed consistently around
36-43\% lower OR for women achieving a complete 1-h sepsis bundle compared to men regardless of mode of ED arrival, age, vital signs, comorbidities, suspected focus or severity. Small differences favoring men were also found in the EMS, but during ICU stay, no differential management was found concerning nurse workload, mechanical ventilation or ICU length of stay.

After risk adjustment, women had 28\% higher odds of death at 30 days, but we found no evidence that the increased mortality was mediated by lower bundle completion. In crude analysis, the mortality difference was most pronounced in patients over the age of 50 .

\section{Differential management}

A previous study of 340 patients with severe sepsis presenting to the ED [33] identified female sex as one factor among several associated with nonadherence to early goal-directed therapy.

Only one previous study [18, 19], has systematically explored the issue of sex and management of critically ill sepsis patients presenting to the ED. Among 814 patients with severe sepsis or septic shock, it was found that men received antibiotics $20 \%$ faster than women (184 vs $153 \mathrm{~min}, p<0.001$ ) [19]. Unlike the present study, it found no significant difference in sepsis bundle completion. The studies differ, however, the previous study was single center, participated in the surviving sepsis campaign (SSC) and had quality assurance teams providing performance feedback. The resuscitation bundle had six elements and a 6-h time frame, whereas the Swedish bundle had just four elements, and a 1-h time frame. The Swedish register is nationwide, multicenter, has no SSC association and only recently has a minority of Swedish hospitals begun to provide regular feedback to physicians regarding time to antibiotics. We believe that our study is representative of the majority of hospitals which are neither SSC associated nor have sepsis quality assurance teams.

Mandated protocols for detection and treatment of sepsis have been associated with improved survival as shown by Seymour [14]. They may also help to equalize management. Seymour found that although 3-h sepsis bundle compliance was higher in men ( 83.8 vs $81.1 \%$, $p<0.001)$, even after introduction of mandated protocols, the relative difference was much smaller than in 

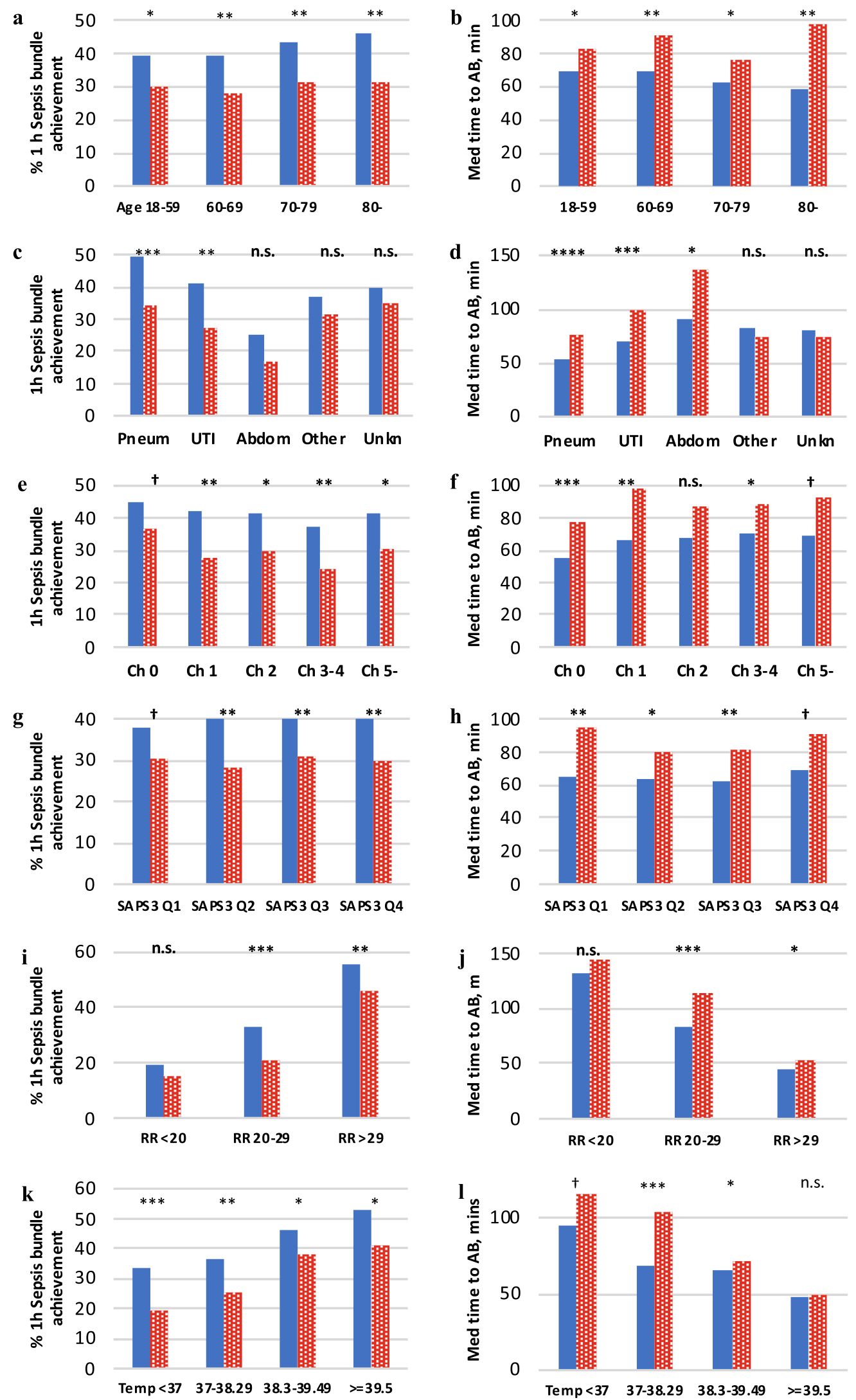
Table 3 Risk factors for 30-day mortality ${ }^{a}$

\begin{tabular}{|c|c|c|c|c|c|c|}
\hline \multirow[t]{2}{*}{ Characteristic } & \multicolumn{3}{|c|}{ Univariate analysis $(n=2720)$} & \multicolumn{3}{|c|}{ Multi-variate analysis $^{b}\left(n=2430^{c}\right)$} \\
\hline & OR & $95 \% \mathrm{Cl}$ & $p$ value & OR & $95 \% \mathrm{Cl}$ & $p$ value \\
\hline \multicolumn{7}{|l|}{ Demography } \\
\hline Sex (female) & 1.11 & $0.91-1.36$ & 0.30 & 1.28 & $1.00-1.64$ & 0.05 \\
\hline Age & 1.04 & $1.03-1.05$ & $<0.001$ & & & \\
\hline \multicolumn{7}{|l|}{ Severity of disease } \\
\hline SAPS3 (per unit increase) & 1.07 & $1.06-1.08$ & $<0.0001$ & & & \\
\hline tSAPS3 (per unit increase) & 1.07 & $1.06-1.08$ & $<0.0001$ & 1.06 & $1.05-1.07$ & $<0.001$ \\
\hline Body temperature, per ${ }^{\circ} \mathrm{C}$ increase & 0.78 & $0.75-0.82$ & $<0.001$ & 0.82 & $0.77-0.87$ & $<0.001$ \\
\hline \multicolumn{7}{|l|}{ Quality of care } \\
\hline $1 \mathrm{~h}$ sepsis bundle achieved & 1.06 & $0.89-1.28$ & 0.51 & & & \\
\hline Incorrect $A B$ & 1.50 & $1.06-2.12$ & 0.02 & 1.73 & $1.13-2.65$ & 0.01 \\
\hline \multicolumn{7}{|l|}{ Treatment limitations } \\
\hline At $48 \mathrm{~h}$ & 10.30 & $8.42-12.62$ & $<0.001$ & 8.06 & $6.42-10.12$ & $<0.001$ \\
\hline
\end{tabular}

$A B$ antibiotic, ED emergency department, ICU Intensive Care Unit, SAPS3 Simplified Acute Physiology Score 3, tSAPS3 SAPS3 minus points for temperature component of score, treatment limitations: any treatment limitations ordered within $48 \mathrm{~h}$ of admittance ${ }^{\mathrm{a}}$ estimated by GEE logistic regression ${ }^{\mathrm{b}}$ adjusted for all variables in the column. 'Minus 290 patients who lacked information on body temperature

our study. Comparison is difficult however, since the study included patients with lower illness severity and for various reasons excluded more than half of patients who were identified from analysis.

In the ICU, we found no evidence of differential management, which contrasts with previous studies which have found less deep venous thrombosis prophylaxis, fewer hemodialysis catheters [9], less invasive mechanical ventilation $[9,20]$, and shorter $\operatorname{LOS}[20]$ in women compared to men. Possible reasons for discrepant results include differences in study sizes, studied variables and case mixes. Concordant with this report, another large Swedish ICU study [10] reported higher nurse workload and longer ICU LOS for men among overall admissions but not in the sepsis subgroup.

\section{Mortality}

Previous studies on sex and mortality among critically ill sepsis patients show conflicting results: some found higher mortality among women $[9,21,22]$ some in men $[20,34]$, whereas others reported no difference $[18,24$, 25]. One study [10] analyzed SIR for sex-based differences in outcome and use of ICU resources in all ICU admissions from 2008 through 2012, and in subgroups including 9830 patients with sepsis (including postoperative and nosocomial infections). Adjusted OR for 30-day mortality from sepsis for women was 1.17 (CI 95\% 1.031.33, $p=0.002$ ). The result was deemed interesting but statistically insignificant (a conservative significance level was set to $<0.001$ due to multiple testing), yet is in line with our findings.
We found no association between mortality and sepsis bundle completion. This study was not designed for a thorough scrutiny of all hospital procedures. Therefore, we lack important information needed for a detailed analysis of the mediators of increased odds of death. Nonetheless, it is possible that unequal treatment reflected in sepsis bundle completion and time to antibiotics also affects other aspects of care not captured in our data, which may have contributed to worse outcomes in women.

\section{Strengths and weaknesses}

Our study has several strengths. First, it is multicenter, is well powered for detection of treatment imbalances, and draws on information from independent national registers for an unbiased nationwide identification of patients with community-acquired severe sepsis and septic shock. Second, models were adjusted for a wide range of potential confounders. Third, results on treatment imbalances are robust and remain stable in subgroup analyses and in multivariable models. A limitation is that the study reflects only Swedish conditions, but since Sweden is ranked among countries with the lowest gender gap and inequality $[35,36]$, conditions may plausibly be worse elsewhere. The study suggests inferior care for female patients, but cannot conclusively demonstrate that this is the case. Residual confounding from factors not recorded in the databases cannot be ruled out.

The register does not include information on patients admitted to hospital wards. This may have resulted in selection bias if some women received equal or better treatment in the EMS or ED and thereby avoided ICU 
admission. However, to our knowledge, there are no studies suggesting that septic women receive superior EMS/ED care or respond better to treatment than men.

The NQSR is based on routine medical records, likely explaining missingness of complete data, particularly on sepsis bundles in 24\% of cases. Missingness has been addressed through careful analysis of existing data and sensitivity analyses. Physiological data used for SAPS3 calculations were complete in $79 \%$ of cases. This compares favorably with a general Swedish ICU cohort, in which $59 \%$ of patients had complete variables, but for which SAPS3 was nevertheless considered to perform well [37].

SAPS3 is calculated on ICU admittance, up to $24 \mathrm{~h}$ after ED arrival, and partly reflects post-resuscitation status-which introduces the risk of adjusting for effects of treatment [38]. However, since omitting the SAPS3 subscores that reflect acute physiological derangement made little difference for the estimates, such a bias likely had a low impact. Even though evaluation of infection was performed by experienced ID specialists, some patients may have been incorrectly diagnosed. Finally, since the NQSR only encompasses hospitals where ID physicians are present, and registration rates vary between centers, it does not capture all eligible patients [26].

\section{Conclusions}

Among critically ill patients with severe sepsis or septic shock, women had lower sepsis bundle completion and longer time to antibiotics in the ED compared to men. Differences were pronounced and consistent in subgroups and in analyses adjusted for potential confounders including severity of disease. There were also small differences favoring men in the EMS, but not in the ICU. In adjusted analyses, women had $28 \%$ higher odds of dying than men, but the increase was not mediated by lower bundle completion. Further studies are needed to explore underlying causes of the observed differences in treatment and outcome, and to examine their occurrence in a general ED population.

\section{Electronic supplementary material}

The online version of this article (https://doi.org/10.1007/s00134-019-05910-9) contains supplementary material, which is available to authorized users.

\footnotetext{
Author details

${ }^{1}$ Division of Infectious Diseases 173 and Center for Infectious Medicine, Karolinska Institutet at Karolinska University Hospital Huddinge, 14186 Stockholm, Sweden. ${ }^{2}$ EPI@LUND, Department of Laboratory Medicine, Lund University, Lund, Sweden. ${ }^{3}$ Department of Clinical Sciences, Section for Infection Medicine, Lund University, Skåne University Hospital, Lund, Sweden. ${ }^{4}$ Department of Infectious Diseases Lund, Skåne University Hospital, Hälsogatan 3, SE-221 85 Lund, Sweden.
}

\section{Acknowledgements}

Open access funding provided by Karolinska Institute. We want to express our deep gratitude to dedicated colleagues throughout Sweden who painstakingly keep the quality registers up to date.

\section{Author contributions}

JSC designed the study, analyzed and interpreted data, wrote the paper, constructed tables and figures and performed literature search. MI contributed to study design, data analysis and interpretation and reviewed the text. AN contributed to data analysis and reviewed the text.

\section{Funding}

JSC is supported by Swedish Government Research Grant and Center for Innovative Medicine (CIMED), Karolinska Institute. MI is supported by an investigator grant from the Swedish Government Funds for Clinical Research (ALF).

\section{Compliance with ethics standards}

\section{Conflicts of interest}

All authors report no conflict of interest.

\section{Ethical approval}

The study was performed according to the ethical standards of the 1964 Declaration of Helsinki and was approved by the ethical review board in Stockholm (2015/901-32). Written informed consent was waived.

\section{Open Access}

This article is licensed under a Creative Commons Attribution-NonCommercial 4.0 International License, which permits any non-commercial use, sharing, adaptation, distribution and reproduction in any medium or format, as long as you give appropriate credit to the original author(s) and the source, provide a link to the Creative Commons licence, and indicate if changes were made. The images or other third party material in this article are included in the article's Creative Commons licence, unless indicated otherwise in a credit line to the material. If material is not included in the article's Creative Commons licence and your intended use is not permitted by statutory regulation or exceeds the permitted use, you will need to obtain permission directly from the copyright holder.To view a copy of this licence, visit http://creativecommons.org/licen ses/by-nc/4.0/.

\section{Publisher's Note}

Springer Nature remains neutral with regard to jurisdictional claims in published maps and institutional affiliations.

Received: 26 June 2019 Accepted: 19 December 2019

Published online: 23 January 2020

\section{References}

1. Ayanian JZ, Epstein AM (1991) Differences in the use of procedures between women and men hospitalized for coronary heart disease. $N$ Engl J Med 325:221-225

2. Rathore SS, Chen J, Wang Y, Radford MJ, Vaccarino V, Krumholz HM (2001) Sex differences in cardiac catheterization: the role of physician gender. JAMA 286:2849-2856

3. Reeves M, Bhatt A, Jajou P, Brown M, Lisabeth L (2009) Sex differences in the use of intravenous rt-PA thrombolysis treatment for acute ischemic stroke: a meta-analysis. Stroke 40:1743-1749

4. Lyratzopoulos G, Abel GA, McPhail S, Neal RD, Rubin GP (2013) Gender inequalities in the promptness of diagnosis of bladder and renal cancer after symptomatic presentation: evidence from secondary analysis of an English primary care audit survey. BMJ Open 3(6):e002861

5. Borkhoff CM, Hawker GA, Kreder HJ, Glazier RH, Mahomed NN, Wright JG (2008) The effect of patients' sex on physicians' recommendations for total knee arthroplasty. CMAJ 178:681-687

6. Gomez D, Haas B, de Mestral C, Sharma S, Hsiao M, Zagorski B, Rubenfeld G, Ray J, Nathens AB (2012) Gender-associated differences in access to trauma center care: a population-based analysis. Surgery 152:179-185 
7. Fowler RA, Sabur N, Li P, Juurlink DN, Pinto R, Hladunewich MA, Adhikari NK, Sibbald WJ, Martin CM (2007) Sex-and age-based differences in the delivery and outcomes of critical care. CMAJ 177:1513-1519

8. Valentin A, Jordan B, Lang T, Hiesmayr M, Metnitz PG (2003) Genderrelated differences in intensive care: a multiple-center cohort study of therapeutic interventions and outcome in critically ill patients. Crit Care Med 31:1901-1907

9. Pietropaoli AP, Glance LG, Oakes D, Fisher SG (2010) Gender differences in mortality in patients with severe sepsis or septic shock. Gend Med 7:422-437

10. Samuelsson C, Sjoberg F, Karlstrom G, Nolin T, Walther SM (2015) Gender differences in outcome and use of resources do exist in Swedish intensive care, but to no advantage for women of premenopausal age. Crit Care 19:129

11. Liu V, Escobar GJ, Greene JD, Soule J, Whippy A, Angus DC, Iwashyna TJ (2014) Hospital deaths in patients with sepsis from 2 independent cohorts. JAMA 312:90-92

12. Ferrer R, Martin-Loeches I, Phillips G, Osborn TM, Townsend S, Dellinger RP, Artigas A, Schorr C, Levy MM (2014) Empiric antibiotic treatment reduces mortality in severe sepsis and septic shock from the first hour: results from a guideline-based performance improvement program. Crit Care Med 42:1749-1755

13. Liu VX, Fielding-Singh V, Greene JD, Baker JM, Iwashyna TJ, Bhattacharya J, Escobar GJ (2017) The timing of early antibiotics and hospital mortality in Sepsis. Am J Respir Crit Care Med 196:856-863

14. Seymour CW, Gesten F, Prescott HC, Friedrich ME, I washyna TJ, Phillips GS, Lemeshow S, Osborn T, Terry KM, Levy MM (2017) Time to Treatment and mortality during mandated emergency care for sepsis. N Engl J Med 376:2235-2244

15. Levy MM, Gesten FC, Phillips GS, Terry KM, Seymour CW, Prescott HC, Friedrich M, Iwashyna TJ, Osborn T, Lemeshow S (2018) Mortality changes associated with mandated public reporting for sepsis. The results of the New York State Initiative. Am J Respir Crit Care Med 198:1406-1412

16. Nguyen HB, Corbett SW, Steele R, Banta J, Clark RT, Hayes SR, Edwards J, Cho TW, Wittlake WA (2007) Implementation of a bundle of quality indicators for the early management of severe sepsis and septic shock is associated with decreased mortality. Crit Care Med 35:1 105-1112

17. Linner A, Sunden-Cullberg J, Johansson L, Hjelmqvist H, Norrby-Teglund A, Treutiger CJ (2013) Short- and long-term mortality in severe sepsis/ septic shock in a setting with low antibiotic resistance: a prospective observational study in a Swedish university hospital. Front Public Health 1.51

18. Madsen TE, Simmons J, Choo EK, Portelli D, McGregor AJ, Napoli AM (2014) The DISPARITY Study: do gender differences exist in Surviving Sepsis Campaign resuscitation bundle completion, completion of individual bundle elements, or sepsis mortality? J Crit Care 29:473

19. Madsen TE, Napoli AM (2014) The DISPARITY-II study: delays to antibiotic administration in women with severe sepsis or septic shock. Acad Emerg Med 21:1499-1502

20. Xu J, Tong L, Yao J, Guo Z, Lui KY, Hu X, Cao L, Zhu Y, Huang F, Guan X, Cai C (2018) Association of sex with clinical outcome in critically III sepsis patients: a retrospective analysis of the large clinical database Mimic-III. Shock 52(2):146

21. Vincent JL, Sakr Y, Sprung CL, Ranieri VM, Reinhart K, Gerlach H, Moreno R, Carlet J, Le Gall JR, Payen D (2006) Sepsis in European intensive care units: results of the SOAP study. Crit Care Med 34:344-353

22. Combes A, Luyt CE, Trouillet JL, Nieszkowska A, Chastre J (2009) Gender impact on the outcomes of critically ill patients with nosocomial infections. Crit Care Med 37:2506-2511
23. Adrie C, Azoulay E, Francais A, Clec'h C, Darques L, Schwebel C, Nakache D, Jamali S, Goldgran-Toledano D, Garrouste-Orgeas M, Timsit JF (2007) Influence of gender on the outcome of severe sepsis: a reappraisal. Chest 132:1786-1793

24. van Vught LA, Scicluna BP, Wiewel MA, Hoogendijk AJ, Klouwenberg PMC, Ong DSY, Cremer OL, Horn J, Franitza M, Toliat MR, Nurnberg P, Bonten MMJ, Schultz MJ, van der Poll T, Consortium M (2017) Association of gender with outcome and host response in critically III sepsis patients. Crit Care Med 45:1854-1862

25. Mahmood K, Eldeirawi K, Wahidi MM (2012) Association of gender with outcomes in critically ill patients. Crit Care 16:R92

26. Svefors J (2017) Årsrapport Svår Sepsis 2016. Svenska Infektionsläkarföreningen. http://infektion.net/sites/default/files/pdf/kvalreg_inf_2016_ sepsis.pdf. Accessed 3 May 2019

27. Sjoberg F, Walther S (2012) Intensive care registries and the evolution of the concept of 'quality of care'-reflections from the 10-year anniversary symposium of the Swedish Intensive Care Registry. Acta Anaesthesiol Scand 56:1073-1077

28. Ludvigsson JF, Andersson E, Ekbom A, Feychting M, Kim JL, Reuterwall C, Heurgren M, Olausson PO (2011) External review and validation of the Swedish national inpatient register. BMC Public Health 11:450

29. Wettermark B, Hammar N, Fored CM, Leimanis A, Otterblad Olausson P, Bergman U, Persson I, Sundstrom A, Westerholm B, Rosen M (2007) The new Swedish Prescribed Drug Register-opportunities for pharmacoepidemiological research and experience from the first six months. Pharmacoepidemiol Drug Saf 16:726-735

30. Bone RC, Balk RA, Cerra FB, Dellinger RP, Fein AM, Knaus WA, Schein RM, Sibbald WJ (1992) Definitions for sepsis and organ failure and guidelines for the use of innovative therapies in sepsis. The ACCP/SCCM Consensus Conference Committee. American college of chest physicians/society of critical care medicine. Chest 101:1644-1655

31. Atzema CL, Austin PC, Tu JV, Schull MJ (2010) ED triage of patients with acute myocardial infarction: predictors of low acuity triage. Am J Emerg Med 28:694-702

32. Sunden-Cullberg J, Rylance R, Svefors J, Norrby-Teglund A, Bjork J, Inghammar M (2017) Fever in the emergency department predicts survival of patients with severe sepsis and septic shock admitted to the ICU. Crit Care Med 45:591-599

33. Mikkelsen ME, Gaieski DF, Goyal M, Miltiades AN, Munson JC, Pines JM, Fuchs BD, Shah CV, Bellamy SL, Christie JD (2010) Factors associated with nonadherence to early goal-directed therapy in the ED. Chest 138:551-558

34. Adrie C, Azoulay E, Francais A, Clec'h C, Darques L, Schwebel C, Nakache D, Jamali S, Goldgran-Toledano D, Garrouste-Orgeas M, Timsit JF, OutcomeRea Study G (2007) Influence of gender on the outcome of severe sepsis: a reappraisal. Chest 132:1786-1793

35. World Economic Forum (2018) Global gender gap report. https://www. weforum.org/reports/the-global-gender-gap-report-2018. Accessed 2 May 2019

36. United Nations Development Programme (2018) Gender Inequality Index report 2018. http://hdr.undp.org/en/content/gender-inequality-index-gii. Accessed 2 May 2019

37. Engerstrom L, Nolin T, Mardh C, Sjoberg F, Karlstrom G, Fredrikson M, Walther SM (2017) Impact of missing physiologic data on performance of the simplified acute physiology score 3 risk-prediction model. Crit Care Med 45:2006-2013

38. Tunnell RD, Millar BW, Smith GB (1998) The effect of lead time bias on severity of illness scoring, mortality prediction and standardised mortality ratio in intensive care-a pilot study. Anaesthesia 53:1045-1053 